\title{
Una temprana psicología de la ciencia en el Paraguay
}

\author{
An Early Psychology of Science in Paraguay
}

\author{
José E. García \\ Universidad Católica Nuestra Señora de la Asunción, Asunción, Paraguay.
}

Psicólogo. Docente universitario. Director de la revista Psicología Básica y Aplicada. Sus áreas de investigación principales son historia de la psicología, formación del psicólogo y psicología evolucionista.

Recibido: $22-03-16$

Aprobado: 20-06-16

*Correspondencia

Email: joseemiliogarcia@hotmail.com

\section{Citar como:}

Garcia, J. (2016). Una temprana psicología de la ciencia en el Paraguay. Propósitos y Representaciones, 4(2), 359-412. Doi: http://dx.doi.org/10.20511/pyr2016.v4n2.125

(c) Universidad San Ignacio de Loyola, Vicerrectorado de Investigación y Desarrollo, 2016. (cx) BY-NC-ND Este artículo se distribuye bajo licencia CC BY-NC-ND 4.0 Internacional (http://creativecommons.org/licenses/by-nc-nd/4.0/). 


\section{Resumen}

La psicología de la ciencia es un campo de investigación surgido a finales de la década de 1980 y cuyo interés fundamental es el estudio de las condiciones que determinan el surgimiento y desarrollo de los científicos e investigadores. Sin embargo, pese a su aparente novedad, es factible encontrar antecedentes diseminados ampliamente en la obra de autores previos. Uno de ellos es R. Ross, quien escribió un artículo en la revista paraguaya Letras en 1915. Ross sostenía que la producción de genios es una de las potencialidades más valiosas a las que puede aspirar una nación y que ella se encuentra en un grado de importancia superior a otra clase de riquezas. En su exposición abunda en consideraciones relacionadas con los procesos subjetivos que conducen la inspiración creadora, la generación de ideas novedosas y las relaciones entre la genialidad y la locura, punto en el que se ajusta a las ideas del médico italiano Cesare Lombroso. La conclusión del artículo es que las ideas de Ross pueden vislumbrarse como un antecedente lejano para la psicología de la ciencia, aunque no haya alcanzado una continuidad posterior en la obra de otros autores paraguayos. La metodología adoptada es a un tiempo descriptiva y crítica, con un análisis contextual de las fuentes primarias que resultan pertinentes al problema.

Palabras clave: R. Ross, psicología de la ciencia, genios, historia de la psicología, psicología en Paraguay.

\section{Summary}

The psychology of science is a field of research emerged in the late 80's and its basic interest is the study of the conditions determining the rise and development of scientists and researchers. However, in spite of its apparent novelty, it is feasible to find background widely disseminated in the work of previous authors. One of them is R. Ross, who wrote an article in the Paraguayan journal Letras in 1915. Ross argued that geniuses' production is one of the most valuable potentials to which a nation can aspire and has a 
relevance degree higher than any kind of wealth. His argument agrees with considerations related to the subjective processes leading creative inspiration, the generation of new ideas and the relations between genius and insanity, a view that fits the ideas of the Italian physician Cesare Lombroso. The article concludes that Ross' ideas may be identified as a distant background for the psychology of science, although it has not reached a later continuity in the work of other Paraguayan authors. The methodology adopted is both descriptive and critical, with a contextual analysis of the primary sources that are relevant to the problem.

Keywords: R. Ross, psychology of science, genius, history of psychology, psychology in Paraguay. 


\section{Introducción}

Hay varias formas como los psicólogos pueden manifestar interés hacia la ciencia. La pertenencia a un dominio que se identifica primordialmente con la producción de conocimientos científicos resultó fundamental para la formación de su identidad disciplinaria y en la consolidación del estatus de la disciplina en cuanto área independiente de experticia. Solo hay que recordar que la psicología, de acuerdo con la reconstrucción de los historiadores más tradicionales (Baldwin, 1913a, 1913b; Brett, 1912; Klemm, 1914; Mercier, 1918; Rand, 1912), proviene del tronco mayor de la filosofía, un campo fundamentalmente reflexivo del que la mayoría de los psicólogos se ha esforzado en tomar cautelosa distancia, como un requisito básico para el reconocimiento pleno de su autonomía.

En un sentido cronológico, las relaciones entre ambos sectores provienen de la antigüedad griega. La filosofía suministró a la psicología algunos de sus marcos de referencia más consistentes y duraderos (García, 2015a), incorporando esquemas teóricos de tal amplitud y profundidad como el dualismo encarnado por Pitágoras y Platón y el monismo que representaron Demócrito y Aristóteles (Uttal, 2004), así como la idea de que los conocimientos o bien son innatos o se aprenden en el contacto diario con la realidad circundante (Fine, 2014), además de otros hitos conceptuales semejantes.

Para autores como Cacciopo y Freberg (2013), el árbol familiar de la psicología incluye por igual a la filosofía y a las ciencias físicas. Desde luego, tampoco es posible marginar a la medicina pues algunos de los grandes personajes que se destacaron en el cuidado de la salud en el mundo antiguo tuvieron algo que decir sobre la naturaleza de la personalidad, el carácter y el temperamento (Dumont, 2010) o pretendieron innovar sobre aspectos prácticos con la aplicación de terapias arcaicas para los problemas que afectan a la mente y el comportamiento (Millon, 2004). Esta variante de la inclinación hacia la ciencia posee connotaciones directas sobre la imagen 
pública que obtienen los practicantes y forman parte del esfuerzo colectivo por delimitar una identificación propia para la disciplina.

Una segunda forma como la psicología logra aproximarse a la ciencia es convirtiéndola en una de sus esferas de interés investigativo, con lo cual se transforma en un elemento nuevo para el análisis y hace posible acceder a una explicación de su dinámica y procesos particulares. Como el objeto de la psicología es el comportamiento y los fenómenos cognitivos, resulta evidente que esa es la dimensión principal hacia la que habrá de orientar su indagación. Quienes optan por esta faceta de la relación entre psicología y ciencia posiblemente evoquen, en primer lugar, a la moderna subdisciplina de la psicología de la ciencia, cuyos primeros trabajos comenzaron a ser difundidos hace relativamente poco tiempo, en los mediados de la década de 1980 (Simonton, 1988).

La gama temática que cubre el actual estudio psicológico de la ciencia es muy extenso y abarca una amplia variedad de materias, como la psicología biológica de la ciencia, la psicología del desarrollo de la ciencia, la psicología cognitiva de la ciencia, la psicología de la personalidad de la ciencia y la psicología social de la ciencia, entre otros ámbitos vinculados (Feist, 2006, 2011). Pero, como todas las ideas influyentes en la psicología, esta nueva subdisciplina no se encuentra desprovista de antecedentes. Feist \& Gorman (2013) señalan a la obra del botánico franco-suizo Alphonse de Candolle (1806-1893) como uno de los predecesores más importantes. Él escribió un libro denominado Histoire des sciences et des savants depuis deux siècles (Candolle, 1873), donde ofrecía una interpretación ambientalista para dar cuenta de las influencias que determinan el surgimiento de las eminencias científicas. Aparte de todos sus méritos, este trabajo fue una reacción al libro de orientación hereditarista que el británico Sir Francis Galton (1822-1911) había publicado unos años antes y que hoy es un clásico de la ciencia, el muy conocido Hereditary genius (Galton, 1869).

Pero Candolle también, de acuerdo con el criterio de Feist \& Gorman (2013), impulsó a Galton a concebir English men of science: Their nature 
and nurture (Galton, 1874), tratado al que habitualmente se considera el primero relacionado con el estudio de la psicología de los científicos. $\mathrm{Y}$ este es un punto fundamental a considerar, ya que autores tan importantes como estos entregaron aportes teoréticos relevantes casi un siglo antes del establecimiento formal del nuevo campo.

Sin embargo, la psicología de la ciencia no es la primera disciplina en concentrarse seriamente sobre estos asuntos sino, por el contrario, la que mostró el surgimiento más tardío. En efecto, existe una filosofía de la ciencia que tuvo su producción más significativa en la obra del austríaco Karl Popper (1902-1994) y del estadounidense Thomas S. Kuhn (1922-1996), el primero de los cuales construyó un esquema teórico coherente y riguroso que permitió un punto de vista intermedio entre el dogmatismo y el relativismo (Gattei, 2009), mientras que el segundo instauró uno de los sistemas más influyentes surgidos en el análisis sobre el desarrollo de la ciencia (Hoyningen-Huene, 1993).

No obstante, debe mencionarse que ya algunos científicos como René Descartes (1596-1650), Isaac Newton (1642-1727) y Albert Einstein (18791955) cumplieron un rol muy significativo para el avance futuro de la filosofía de la ciencia (Okasha, 2002), ya desde muchos siglos antes inclusive. Varios acontecimientos se asociaron para el establecimiento formal de esta subdisciplina (Feist, 2012), entre ellos la publicación de la famosa obra del matemático francés Henri Poincaré (1854-1912), La ciencia y la hipótesis, en 1908 (Poincare, 1918), y la fundación, en 1910, del Círculo de Viena, un selecto y exigente club de filósofos y científicos, al que Lang (2007) llamó "un único vórtice de ideas".

Por otra parte, la historia de la ciencia, que ya posee antecedentes remotos en las obras de autores griegos y sirios, encuentra sus precursores en las modernas academias de ciencia europeas que comenzaron a reunirse en el siglo XVIII. Su fundador moderno es el químico belga George Sarton (18841956), al que se considera el iniciador del estudio histórico de la ciencia por sus numerosas publicaciones (Sarton, 1948) y por la introducción de estos 
temas en el ámbito académico. Fue, además, el fundador de la revista Isis en 1913. De igual modo, existe una sociología de la ciencia que, aunque encuentra un pilar fundamental en la obra de William Ogburn (1886-1959) Social change, de 1922, tuvo su impulsor principal en Robert K. Merton (1910-2003), cuyas numerosas obras ayudaron a consolidar la reunión entre ciencia y sociología.

Concebida en términos modernos, la psicología de la ciencia es un espacio de indagación sumamente fructífero que posee nexos e implicancias con muchos otros campos. Entre ellos, por ejemplo, la psicología educacional constituye un vértice singularmente interesante. Esta se define como la ciencia del comportamiento humano aplicada a la enseñanza y los procesos de aprendizaje (Tuckman \& Monetti, 2011). Su propósito es generar conocimiento práctico encaminado a solucionar comportamientos que asoman en los contextos educativos. Es, por consiguiente, un ámbito destinado esencialmente a la investigación.

Siendo la psicología educacional un territorio que se identifica con la profesión aplicada, no cabe duda de que el estudio de los eventos sociales e individuales que conducen al surgimiento de individuos altamente calificados, y que en circunstancias óptimas incluso podrían encaminar sus aptitudes a la creación de nuevas teorías científicas o tecnológicas que supongan grados remarcables de innovación, es -o debería ser- una parte esencial para los objetivos insertos en la enseñanza escolar. De hecho, varios de los fundadores históricos de la psicología de la educación investigaron el modo en que diversos ambientes generan condiciones apropiadas para el aprovechamiento de individuos excepcionalmente dotados (García, 2015b).

El descubrimiento de las vicisitudes familiares y personales que cabe estimar como antecedentes directos o mediatos para la aparición del genio científico no solo interesó a autores clásicos como Galton, sino que también conforma uno de los sustratos actuales de la psicología y la educación de los niños talentosos o superdotados (Simonton, 2014). Además, la manera en que la educación y el entrenamiento que brinda la escuela afecta el desarrollo 
creativo en la ciencia, y el modo en que el rol de los maestros puede maximizar la eficacia de estos procesos, se encuentran en el corazón mismo de la agenda para la psicología educacional (Simonton, 2009). Estos mismos fenómenos pueden llevarnos a comprender por qué otros seres humanos, menos afortunados por cuanto les toca como dotación natural, se hallan tan alejados de las reales posibilidades de acceder a logros de relevancia en tales menesteres. Los puentes que unen a la psicología de la ciencia y los científicos con los actuales desafíos enfrentados por la psicología educacional son más amplios de lo que a primera vista parecen y apenas comienzan a ser vislumbrados.

El que un área de estudios novedoso y emergente, como es hoy la psicología de la ciencia, deba justificar unos antecedentes situados en algún periodo temporal anterior no obedece a una ley histórica de hierro, por lo que cabría admitir excepciones a su pretendida vigencia. No obstante, sí resulta muy probable que puedan hallarse expresiones previas en la obra de autores precedentes que anticiparon algunas de las posiciones defendidas en nuestros días. Esto se debe a que los conocimientos no emergen de la nada ni surgen por generación propia, como sería el caso con las creaciones generosas de alguna deidad ociosa. Por el contrario, las ideas científicas siguen un curso propio que le debe mucho a la evolución de las mismas sociedades que albergan a sus creativos circunstanciales, así como de los procesos internos propios de cada disciplina y las dimensiones muy dinámicas que se ponen en juego en la compleja relación entre sujeto y objeto.

En este sentido, el estudio de la historia de la psicología resulta muy útil y beneficioso ya que permite situar y analizar bibliografía remota que podría resultar factible de considerar en una relación de precedencia temporal con el pensamiento contemporáneo. Su identificación precisa no se realiza con el deseo de erigir una interpretación celebratoria de las personas y los eventos involucrados, sino de comprender, en una manera más completa y acabada, la lógica básica que rige las transformaciones del conocimiento especializado. De hecho, las modernas teorías explicativas sobre los procesos 
que conducen a la evolución de las teorías, o las posibles continuidades o disrupciones que se manifiestan en las mismas, toman en cuenta tanto los factores que operan desde el interior como desde el exterior de la psicología, y solo varían en el peso relativo que conceden a cada uno de estos elementos (Pérez Ríos, 1990).

Apoyados en esas consideraciones, los propósitos que orientan este artículo son: a) Examinar un ensayo del autor R. Ross en el año 1913 y publicado en las páginas de la revista paraguaya Letras como una posible muestra de una preocupación temprana en los temas que hoy se incluyen como parte de la psicología de la ciencia, b) Comparar los puntos de vista defendidos en ese escrito con algunas concepciones que prevalecen en el estudio moderno de la psicología de los científicos, c) Analizar el artículo de R. Ross en el contexto del conocimiento científico y epistemológico vigente a comienzos del siglo XX, y d) Evaluar la importancia del artículo en referencia a la evolución particular de la psicología paraguaya. La metodología que sustenta el estudio es un análisis cualitativo y reposa en el examen e interpretación de las fuentes primarias, en procura de una contextualización apropiada del problema y de las explicaciones que el autor ofrece para una acabada comprensión del mismo.

\section{Un artículo de R. Ross}

El estudio histórico de la psicología paraguaya sugiere varias posibles aristas, una de las cuales es el análisis de su contenido teórico en función de la producción que tuvieron las revistas culturales cuya difusión se inició en el tramo final del siglo XIX y los comienzos del siglo XX. Recientemente, un par de investigaciones se enfocaron en dos de ellas: a) la Revista del Instituto Paraguayo, publicada entre 1896 y 1907 (García, 2014c) y b) Letras, que se editó en 1915 y 1916 (García, 2015c). Estos trabajos no se proponen examinar el contexto cultural más amplio y general en el que cabría insertar su contenido y distribución temática, sino el más específico de las vinculaciones con el modelamiento temprano de la psicología y la 
individualización de los artículos albergados en sus páginas, que además pudieran encerrar un interés concreto para la disciplina.

En el caso de Letras, los escritos comprendidos abarcan cuatro trabajos cuyos enfoques guardan relaciones de afinidad con la psicología. Ellos son los de Báez (1915), Domínguez (1915), Ingenieros (1915a, 1915b) y Mercante (1915). De estos cuatro autores, Báez y Domínguez eran paraguayos, mientras que Ingenieros y Mercante fueron ciudadanos argentinos. Entre estas publicaciones con eventuales resonancias psicológicas, no obstante, hubo una quinta que no fue integrada a la discusión previa de García (2015c). Es un ensayo que se inscribe en una temática de orden más interdisciplinario, adentrándose con preferencia en el dominio de la epistemología de la ciencia aparte de la psicología propiamente dicha.

Sin embargo, como los intentos de clasificación de las ideas y sus afiliaciones o raíces intelectuales no siempre resultan muy directos ni enteramente claros de discernir, cabe mencionar en beneficio del artículo que, de todas maneras, algunos conceptos utilizados bordean de lleno el perímetro de la psicología. Es más, ciertos puntos en su enfoque general podrían considerarse análogos a los mantenidos modernamente por los psicólogos de la ciencia. En esta forma de arqueología del conocimiento que es la historia de la psicología, corresponde brindar una atención algo más detenida a este artículo y sus coincidencias, declaradas o implícitas, con la psicología de la ciencia bajo su conceptualización actual.

El artículo de R. Ross, titulado El genio de la ciencia, fue publicado en 1915 en el primer número de la revista Letras (Ross, 1915). El responsable más característico de esta publicación, si bien no el único, fue el profesor Manuel Riquelme (1885-1961), uno de los artífices más reconocidos de la psicología paraguaya durante la primera mitad del siglo XX (García, 2014a). Riquelme tiene su propio bien ganado sitial en la historia de la psicología paraguaya, entre otros motivos por la publicación de un importante libro introductorio en 1936 (Riquelme, 1948), el primero con este carácter elaborado por un autor paraguayo. 
El escrito de Ross comienza con una afirmación directa y contundente: la producción de genios es la primera facultad que posee una nación, y ella se encuentra por encima y goza de más importancia que el poseer un suelo fértil, riquezas materiales abundantes o las oportunidades que brinda el comercio. Mayor, incluso, que ostentar un pueblo honesto y dedicado al trabajo. Ello ocurre porque la historia de un país cualquiera es la misma que sus genios, tanto los grandes como los pequeños. Y el que muchas de ellas no hayan encontrado un lugar destacado en la historia moderna se debe, precisamente, a su orfandad de individuos sobresalientes. Carece realmente de importancia el que muchas veces, yuxtaponiéndose al resto de los mortales, ellos reciban solo la desconfianza o la burla general de las demás personas, que no aquilatan sus mismos talentos y capacidades. Por ello, las grandes genialidades constituyen una suerte de preciado y elusivo tesoro que, por lo frágil e infrecuente, resultan más valiosas aún.

A renglón seguido, proclama Ross (1915) que es aquella chispa o fogonazo de la inteligencia lo que determina el instante preciso en la proyección de la inspiración científica. Es parte de la visión convencional de la creatividad que sus movimientos obedecen únicamente a cuanto algunos denominan los momentos eureka, que usualmente se describen como un solo destello de iluminación cegadora (Lee, 2002). En la literatura contemporánea, de hecho, la creatividad misma se asocia con frecuencia con esta experiencia del insight, entendido como el momento de la irradiación en el proceso creativo (Simonton, 1999a). Es admisible, desde luego, que se produzcan intervalos de profundos fogonazos y claridad cuando aparecen esos instantáneos flashes. Con ellos no solo emergen las nuevas ideas sino, además, se transforma toda la visión sobre el problema considerado en el momento.

Pero otros autores sugieren que el fenómeno de la creación o la innovación conceptual es parte de un proceso lento y orgánico que ocurre sobre una base más gradual. El psicólogo Mihaly Csikszentmihalyi opina que el insight se estudia de una mejor manera a través de ciertos procesos mentales que resultan en productos creativos (Csikszentmihalyi, 2014). 
Pero Ross (1915), en cambio, sostenía que tal condición no era enteramente suficiente. Creía que, además, era imprescindible que el sujeto estuviera plenamente convencido de la utilidad de su proyecto creativo. Por lo tanto, era necesario un cerebro con las condiciones de alcanzar una valorización más adecuada de las cosas.

Apoyado en la terminología típica de Gustave Le Bon (1841-1931) en los comienzos del siglo XX, afirmaba que las masas no se interesan en las grandes realizaciones, como pueden ser las que afloran en el dominio del pensamiento, si al mismo tiempo no les proporcionan beneficios obvios e inmediatos, como el alimento, la fortuna o el buen pasar. Aquellos individuos que se hallan distantes de la brillantez, más que prodigar beneficios para el avance de la humanidad, solo imponen sus intereses al resto de los mortales $y$, por ello, no resulta extraño que les acarreen perjuicios antes que favores.

Ross (1915) abunda en reflexiones concernientes a los seres humanos y a aquello en lo que radica la grandeza. En tal sentido, diferencia al hombre que sólo es hábil y capaz del que "puede hacer grandes cosas". Al primero le admite la posibilidad de ser grande en lo pequeño, pero al segundo le está reservada la facultad de ser grande en lo grande. Asociada a todo ello está la facultad de la inteligencia, que se relaciona con sus aspiraciones vitales. Cuando el hombre es joven, elige las cosas a las que habrá de abocarse de acuerdo con la capacidad mental que posee, y esta condición le resulta intransferible. Si su inteligencia es muy baja, entonces sólo aspirará al placer y a las cosas sencillas; si está un poco mejor dotado, irá tras las riquezas y la fama, siempre buscándolas para sí mismo, y si la naturaleza le ha conferido incluso un poco más de capacidad, posiblemente deseará proveer un beneficio a su patria. Finalmente, y si llega al nivel más elevado, su mira estará puesta en el bien de la humanidad, sin tener en cuenta su propia persona, sino el desarrollo de los demás.

A primera vista, la decisión acerca de quiénes fueron los más grandes hombres de la historia podría tornar difícil la elección entre personajes como Isaac Newton (1642-1727), William Shakespeare (1564-1616) o Napoleón 
Bonaparte (1769-1821). De este último podría decirse que fue como un rayo fulgurante pero momentáneo, y que hoy, a gran distancia del tiempo que lo vio cimentar su influencia sobre los hombres, queda poco más que un recuerdo, no superior a los otros dos nombrados. Pero aquí Ross, una vez más, recuerda que Napoleón sólo trabajó para sí mismo, no para el beneficio de alguien más. Shakespeare, en cambio, proyectó su aporte a la humanidad entera clarificando los umbrales más profundos y densos de la conciencia humana a través de su creación literaria, y brindó a la posteridad algo mucho más grande: una lección profunda e imperecedera, un presente incomparablemente mayor, para decirlo con las palabras de Ross (1915). Literatura imbuida de psicología, las condiciones anormales de la mente atrajeron la atención diligente de Shakespeare, quien la convirtió en su tema favorito de estudio (Bucknill, 1859).

Dice el autor que la centella de donde irradian las ideas originales brota espontáneamente en el cerebro de los grandes hombres y hace que se anide en ellos una sensación clara sobre la importancia y el valor de los esfuerzos realizados. Esto los diferencia de los demás individuos, que solo operan en función de sí mismos, siendo así la causa y, a la vez, el destino de sus beneficios. Pero estas condiciones, con ser muy necesarias e imprescindibles, no bastan para alcanzar los más elevados fines. El hombre de ciencia, sostiene Ross (1915), debe contar con la necesaria determinación y decisión, y con el valor de superar los obstáculos que se entrecrucen en su camino. Es decir, no se conjugan solamente algunas condiciones cognitivas; también entran en concurso otras variables que apuntan a los factores emotivos y volitivos. Avanzar en el progreso científico es un camino lleno de vacilaciones y dudas que, además, está franqueado por numerosas dificultades. Por ello, el ascenso de los grandes descubridores y artistas está plagado de fatigas, de perplejidades que surgen al pensar en el valor de sus propias fuerzas y en las posibilidades de llegar adelante.

El triunfo del científico es un equilibrio de las cualidades genuinas que lo adornan, como el genio para concebir ideas, la agudeza para percibir 
donde están los verdaderos problemas, la decisión de lograr lo que se busca y la energía puesta en el trabajo, aunque también depende de encontrar la oportunidad precisa. Aquí, el azar juega su parte, y las condiciones exteriores colocan los ingredientes que faltan. En la historia de los siglos previos abundaron, sin dudas, otros grandes individuos con el mismo o tal vez superior potencial de Newton o Shakespeare, pero no se conjugaron en su camino las ocasiones eficientes para desarrollar a pleno sus potencialidades. Cuando ese elemento, tan esquivo e impredecible, no se presenta, entonces no ocurren las condiciones ideales que potencien el genio. Es, con mucho, una cuestión de azar, tanto como de talento real.

Esto no constituye un menosprecio a las cualidades personales del científico. De hecho, la psicología actual establece con bastante precisión las interconexiones que pueden darse entre las variables de la personalidad y la creatividad. Feist (1998), por ejemplo, llevó a cabo un metaanálisis de numerosas investigaciones y encontró que, en general, las personas creativas se distinguen por estar más abiertas a las nuevas experiencias, ser menos convencionales y concienzudas, exhibir mayor autoconfianza y autoaceptación y dejarse conducir con facilidad. Asimismo, se muestran ambiciosas, dominantes, hostiles e impulsivas. La personalidad genéticamente basada y los factores de la inteligencia pueden dar cuenta de entre el 13 y el 29 por ciento de la variación en el talento científico (Feist, 2010). Los creativos son individuos menos conformistas, y el obtener la aprobación de los demás no les resulta una necesidad indispensable (Owens, 2009).

De acuerdo con el punto de vista de Ross (1915), los hombres dotados de las cualidades necesarias para los grandes logros son los que mejor pueden usufructuar las coyunturas ocasionales que surgen a su paso. Al respecto afirmaba que, "en realidad, las circunstancias favorables son comunes, pero el genio es raro y, hasta cierto punto, él crea las oportunidades" (Ross, 1915, pp. 34). El que todas las condiciones necesarias para desembocar en la producción de los talentos científicos sean infrecuentes es la razón principal, argumentaba Ross (1915), por la que el progreso humano se muestra tan lento, dificultoso y esquivo. 
En realidad, la producción constante de genios e individuos talentosos depende mucho de las aleatoriedades que encierra el ambiente y de un sinfín de variables concurrentes, por lo que no es un factor que surja de manera indiscriminada y repetida en cualquier momento que se considere. El que los genios requieran de unas condiciones culturales y políticas adecuadas para establecerse en un lugar y una época determinados es algo que se halla bien establecido en la moderna literatura psicológica (Simonton, 1999b). También las interrelaciones entre la disponibilidad de sujetos talentosos y el desarrollo global de un país. Lynn y Vanhanen (2002) indican que las naciones cuyas poblaciones poseen grados más elevados de inteligencia probablemente tendrán mejores niveles de logro en lo que concierne a sus actividades educacionales y, probablemente, mayor cantidad de individuos que realicen contribuciones de importancia al bienestar nacional.

Ross (1915) coincidía en que las naciones producen genios de todas clases, aunque solo en determinadas épocas. En contrapartida, hay periodos muy largos de tiempo en que sus respectivas sociedades se encuentran huérfanas de talentos y en que los avances parecen nulos. Esto significa permanecer mayormente estáticos y sin ninguna clase de progreso, el cual no puede producirse en una sociedad cualquiera si esta no está dotada de individuos inquisitivos. Cuando de pronto los talentos empiezan a surgir en una colectividad, será dable esperar que alguna transformación haya comenzado a producirse en el entorno inmediato, pues el nacimiento de personas excepcionalmente capacitadas sugiere, en opinión de Ross (1915), la vigencia de alguna ley biológica que los estimule.

En esta empresa colectiva que es la ciencia, sin embargo, hay espacio para otras personas, con dotaciones intelectuales presuntamente modestas, pero de las que aquella requiere para completar muchas de las tareas esenciales. Esto presupone que hay otra clase de hombres, menos cubiertos de méritos, pero más comunes que los privilegiados candidatos al genio. Son los que trabajan en las faenas de la observación y recolección de los datos básicos, sin apuntar a las grandes síntesis ni teorizaciones o a las grandes construcciones 
de sistemas. Los hombres que se mueven a este nivel del trabajo científico, argumentaba Ross (1915), tal vez no se encuentren provistos de inclinaciones tan brillantes, aunque posean habilidades igualmente útiles y relevantes para los quehaceres de la ciencia: el deseo íntimo de llegar a una obra importante, la plena decisión de realizarlo y la imprescindible virtud de la paciencia. Y teniendo en mente, además, la probabilidad de que nunca llegue una recompensa adecuada y merecida, en justa proporción a la dedicación y el esfuerzo. Por este camino, quizás por vía de la casualidad o del azar caprichoso, puede también arribarse a la obtención de hallazgos oportunos.

La ciencia no es únicamente la consecución exitosa de los descubrimientos logrados por mentes poderosas en el análisis de la realidad y munidos de una inspiración prodigiosa, algo que en la historia de la psicología se asocia a la cuestionada visión de los grandes hombres como únicos artífices del avance científico (Hilgard, Leary \& McGuire, 1991). Los individuos ordinarios, que no llegan a la tempestuosa elevación del genio, pero que con un trabajo tesonero, y sobre todo con el aliado oportuno de la casualidad o la serendipia, consiguen avances importantes, también cumplen un rol singular.

Empeñado en reconocer las clases de individuos en relación con el tipo de actividad que desarrollan en la ciencia, Ross (1915) distingue dos tipos de "cerebros": a) uno que, según nos dice, actúa principalmente como un solucionador de problemas y b) un segundo que, básicamente, es un observador atento de los hechos externos. Ambas tareas son importantes en la dinámica de la investigación, pero tienen alcances diferentes. Para especificar la correspondencia, nuestro autor aseguraba que en la ciencia hay nueve partes de pensamiento y una sola de observación. A todas luces, una proporción acusadamente desigual. En esta perspectiva, la función teórica y analítica aparece por encima de la dimensión práctica, cuya materialización más elemental es la compilación de los datos.

Las diversas contingencias que convergen para el surgimiento del genio hacen propicio el análisis de muchos problemas diferentes aunque relacionados, uno de los cuales es el del vínculo con la locura. Este modo de 
encarar a los individuos excepcionalmente dotados, y de observar de cerca su relación con el comportamiento inusual, lo excéntrico y mórbido, es una de las vertientes principales que han tomado muchos estudios del tema. Ross (1915) tampoco los ignora en su artículo, enfocándolos desde el punto de vista de Cesare Lombroso (1835-1909), el célebre criminólogo italiano que también había estudiado los contextos particulares que sugiere esta esquiva y controversial condición.

De hecho, Lombroso (1891) sugirió que el parecido entre la insania y el genio, aunque no pueda sostenerse que ambos necesariamente se confunden, sirve para demostrar que el uno no excluye al otro, especialmente cuando el mismo sujeto es considerado. Sin mencionar a los individuos excepcionalmente dotados que, en algún momento de sus vidas, sufrieron de alucinaciones o enfermedades mentales, o los que culminaron gloriosas carreras en opacos arrebatos de locura. Como sucedió con Giambattista Vico (1668-1774), por ejemplo, muchos hombres de grandes condiciones intelectuales se vieron sujetos a episodios de monomanía o alucinaciones. Sus detallados análisis de numerosas e insignes figuras de la ciencia y el arte de su tiempo lo llevaron a concluir que, pese a las objeciones que anticipó respecto al alcance de sus conclusiones y al escepticismo que despertó el uso de la estadística como regla y criterio para la interpretación de sus estudios, tales manifestaciones de la locura podrían verse atañidas y aun condicionadas por una serie de circunstancias accidentales, enteramente independientes de la condición física o mental de cada persona.

También otros requerimientos para el trabajo científico exitoso, entre ellos la vigencia de situaciones óptimas que conducen al descubrimiento y la invención, pueden verse subordinados a influencias exteriores precisas. Así adquieren una importancia fundamental los contextos climáticos, que influencian el éxito o el fracaso de los científicos, en formas no siempre evidentes. Los naturalistas, por ejemplo, disfrutan de mayores facilidades para la investigación y el experimento cuando trabajan en los días de clima cálido, mientras que para los anatomistas son particularmente favorables 
las noches frías del invierno, aunque ciertamente los cuerpos humanos puedan ser utilizados en todas las estaciones. Los astrónomos, por otra parte, también obtienen mucho provecho para sus mediciones estelares en las horas nocturnas invernales, cuando la influencia de la refracción es menor.

Decía además Lombroso (1891) que estas circunstancias accidentales afectan otros aspectos aún más cruciales, incluso la muerte, el nacimiento o el asesinato de otros individuos. La importancia de la relación emerge claramente cuando la estadística se incorpora sistemáticamente al contexto explicativo. A la larga, todos conducen a idéntico resultado y a la deducción de un determinante común, el cual solo puede ser encontrado en causas meteorológicas. Tanto las creaciones artísticas como los descubrimientos científicos se hallan asociados entre sí pues sustentan momentos de excitación y extrema sensibilidad en el individuo y fusionan los actos más diversos en fructificadoras síntesis. Estas formas de exaltación consiguen dar fuerza a los instantes de gran fecundidad creadora.

Lombroso pensaba que factores adicionales como la raza y la herencia biológica guardaban estrecha relación con la locura. Su aproximación a estos problemas se fue estructurando paulatinamente en base a las teorías que eran corrientes en su época. Pero su concepto de lo insano era manifiestamente amplio, ya que estaba referido a cualquier desviación observable respecto al comportamiento promedio, algo que no es compartido por los psiquiatras contemporáneos. Como quiera que sea, para Ross (1915) la condición de genialidad era la de una tiranía que actúa sin pausa sobre el individuo. La compara con un fuego ardiente que empuja y, a la vez, devora. Para el conjunto de sus realizaciones, el genio se alimenta de todas las energías internas del hombre. La dotación excepcional se impone y sobrepone a todas las circunstancias adversas que pretenden bloquear su camino, ya sean las actitudes escépticas que emanan del entorno, las críticas, las incomprensiones y hasta el odio o los celos que nacen de los adversarios. En su esencia, es persistente y no se detiene hasta alcanzar el éxito. Este impulso se encuentra anclado en lo profundo de las motivaciones humanas, y atiza su fuego enardecido cual combustible inagotable. 
Ross (1915) toma varios ejemplos de la historia para ilustrar su punto: Sócrates, que debió beber la cicuta; Bruno, que enfrentó el desenfrenado espanto de la hoguera; la persecución y humillación que lanzaron contra Galileo. No acaban las muestras en que los grandes heraldos de la humanidad, sus mentes más preclaras y sus benefactores ilustres, muchas veces, demasiadas, debieron pagar con duras penas su condición de seres más aventajados y proteicos. La incomprensión hace que se difunda la versión de que los genios están locos, o que su trabajo es innecesario y aun perjudicial. Y sobre ellos centra su reflexión final nuestro autor:

Casos análogos han ocurrido recientemente y seguirán ocurriendo. La mancha, lo insano, no está en el cerebro del genio; está en el cerebro del público (Ross, 1915, pp. 35).

Es un hecho lamentable que la humanidad, en términos genéricos, no haya puesto el esfuerzo, el empeño ni la decisión necesarios para favorecer el nacimiento de los genios y permitir su desarrollo y multiplicación. En cualquier situación que haya coartado y suprimido el surgimiento de los mismos o inhibido su florecimiento temprano, los pueblos y su cultura conocieron el rumbo cierto de la decadencia. Decía Ross (1915), de forma un tanto admonitoria, que la tarea fundamental del género humano deberá ser, en las décadas próximas, conservar a los genios o los individuos de inteligencia superior como uno de los tesoros a preservar para las generaciones futuras.

\section{Conclusión}

La psicología de la ciencia es un campo de investigación relativamente nuevo cuyos primeros congresos, sociedades y publicaciones especializadas comenzaron a conocerse en las últimas tres décadas, pero cuyos antecedentes pueden buscarse en la obra de autores diversos que escribieron y teorizaron en el curso de los decenios precedentes. En la actualidad, esta área se ha venido desarrollando con fuerza creciente al interior de la psicología como una vertiente orientada a entender, de una forma sistemática y congruente, las diferentes condiciones biológicas, filogenéticas, ontogenéticas, familiares, 
educacionales, sociales y culturales que desembocan en el surgimiento de individuos creativos en los diferentes ámbitos de la actividad humana, incluyendo el amplio rango de la investigación científica.

En este sentido, la indagación sobre los procesos atinentes a la generación de grandes talentos posee connotaciones prácticas importantes, aunque quizás no muy evidentes todavía, que podrían sintetizarse en la comprensión, desarrollo y sostenimiento de entornos óptimos o, cuando menos, amigables al surgimiento de personas con la capacidad, visión y determinación necesarias para contribuir al desarrollo fructífero de sus propios colectivos culturales.

El desarrollo potencial de individuos altamente creativos tiene importancia para un gran espectro de situaciones, y es innegable que también guarda un componente esencial para cualquier proyecto que tenga como objetivo el desarrollo nacional (García, 2014b). De igual manera, es un campo de conexiones muy prometedoras con sectores especializados, como la psicología de la educación, apoyado en el conocimiento de los efectos que juegan el ambiente del hogar y de la escuela en el establecimiento de invenciones novedosas, y de las estrategias más efectivas para potenciar la inteligencia y la creatividad en los seres humanos mejor dotados.

Las ideas forjadas en el presente registran antecedentes en el pasado. Están representadas en autores que, aunque no hayan albergado la pretensión de anclar una psicología de la ciencia en sentido contemporáneo, adelantaron conceptos y puntos de vista que suenan provocativamente semejantes a las opiniones actuales. El artículo de R. Ross que hemos sometido a un conciso análisis en estas páginas constituye un caso significativo. De una forma clara y terminante, expresó que los genios son parte indisputable de la riqueza nacional de un país, una concepción que se hallaba muy en armonía con los conceptos esgrimidos en la misma época por figuras como el estadista británico Arthur James Balfour (1848-1930), quien sostuvo que algunas variedades de genios contribuyeron al desarrollo social en épocas muy localizadas de la historia, como es el caso de Atenas en los siglos V y IV 
antes de Cristo, de Florencia en los siglos XV y XVI y de Holanda en los siglos XVII y XVIII, por citar unos pocos ejemplos (Balfour, 1908).

La presencia o ausencia de individuos de genio puede incluso comprometer las posibilidades de un país de figurar con algún real merecimiento en las páginas de la historia. Las ideas de Ross representaron la incorporación activa de conceptos psicológicos a una visión de la inteligencia y su contraparte directa: la creatividad. De este modo subrayó aspectos sustanciales, como la motivación que anticipa los logros de aquellos sujetos con mayor capacidad intelectual, los fulgores que anteceden o son la causa para la creación de ideas innovadoras y los rasgos que confieren la grandeza a ciertas personas para distanciarlas agudamente de los simples aprovechadores de circunstancias. Con su defensa de estas ideas, bordeaba directamente los ámbitos de la personalidad y la inteligencia.

Igualmente, Ross (1915) reconoció la equivalencia entre la inteligencia y la grandeza intrínseca de los hombres, poniéndolas en relación con la calidad de sus logros y su mayor o menor proyección hacia el bien de la humanidad entera. Por ello, en los individuos más brillantes destacan siempre las realizaciones orientadas al bien común, mientras que los pequeños solo reconocen la presencia de sus propios y diminutos intereses personales. Comprendió que en el proceso creativo no cuentan solo las aptitudes intelectuales, sino que convergen, además, las emociones y la voluntad de avanzar. En ausencia de ellos, cualquier logro eventual continuará ausente.

También merecieron una atención destacada los contextos ambientales en que surgen los talentos, que son tanto culturales como personales, $\mathrm{y}$ se combinan con la presencia de una determinación y convencimiento intrínseco suficientes para seguir. No obstante, en todas las instancias de avances científicos genuinos se impone el fino equilibrio que proviene de las cualidades subjetivas y cognitivas del científico. Las personas mejor dotadas tienen la capacidad de reconocer con mayor facilidad y rapidez los momentos y las situaciones para explotar las oportunidades ocasionales, los contextos para la invención y el descubrimiento. Ellos 
saben aprovecharlas de mejor forma y con mayor eficiencia que los demás. Basado en estas consideraciones, Ross (1915) pudo distinguir que existen individuos mejor equipados para las tareas elementales, como la recolección y obtención de los datos básicos (el observador de los hechos externos), y los que apuntan a la teorización y el desarrollo de hipótesis de nivel superior (el solucionador de problemas).

Aunque la diferencia pueda sugerir una forma de encubierto elitismo intelectual, es suficiente para atestiguar el reconocimiento de fenómenos como los que hoy denominamos estilos intelectuales (Sternberg, 1997; Zhang \& Sternberg, 2009), sus variantes esenciales y la acción diferenciada que ejercen en el trabajo del investigador. Todos los faroles alumbran en la oscuridad, pero aquellos que hayan sido cargados con mayor provisión de combustible extenderán la luminosidad de su llama un poco más lejos, recortando las sombras del medio nocturno. Donde las lámparas iluminan el terreno, las diferencias en el intelecto encienden los factores que componen el pensamiento. Una metáfora semejante es la que puede extraerse de las ideas de Ross, quien tampoco estuvo lejos de la tendencia de su época, frecuente en autores como Lombroso, de conectar un elevado desarrollo de la inteligencia y la genialidad con una pizca de locura.

Con todo, y pese a su importancia y novedad, el artículo de Ross (1915) constituye solo una aparición fugaz que no dejó influencias reconocibles en la psicología paraguaya ni a corto ni a largo plazo. Su publicación, incluso, puede verse como circunstancial tomando en consideración la obra del mismo autor, sobre el que no se localizan profundizaciones ulteriores. Sin embargo, esta no es una limitación que deba circunscribirse únicamente a este artículo, pues se alza como un rasgo común y frecuente en el contexto de muchas producciones científicas locales. En la historia de la psicología paraguaya, la discontinuidad y la ausencia de tradiciones se evidencian con mayor frecuencia que la consecución exitosa de esfuerzos sostenidos y congruentes (García, 2007, 2009). 
Cabe apuntar que el artículo de Ross tiene la particularidad de anticipar ideas y enfoques concernientes a la personalidad y las motivaciones de los científicos varias décadas antes de su inserción formal en la temática de la ciencia psicológica, incluso tomando en cuenta el escenario internacional. Pero, en el plano local, sus ideas fueron como notas de una melodía ejecutada para oídos que no se encontraban habituados ni adiestrados para escucharla. No es que hubiera completa ausencia de inteligencias interesadas en el cultivo de la ciencia, pero su desarrollo y práctica eran infrecuentes en la cultura nacional de comienzos del siglo XX, dejando a las mentes inquisitivas libradas a una labor solitaria y con escaso reconocimiento social.

El trabajo de Ross (1915) es una pieza típica de la psicología paraguaya del periodo preuniversitario: sólida, bien informada, sobriamente escrita, pero esencialmente teórica. El artículo contenía numerosos elementos de análisis sobre los temas enfocados, algunas posibles fuentes de hipótesis contrastables e indicios para avances teóricos incipientes. Aun así, no condujo a un programa de investigación empírica sostenible.

Nos encontramos frente a lo que ha sido una constante para la historia de la psicología en este país, traducida en el perfil de una especialidad que a veces genera ideas por sí misma y que, con mayor frecuencia, reproduce conocimiento importado o en algún proceso de recepción, pero se frustra en su tránsito del pensamiento y la teoría a la comprobación o la innovación empírica. No obstante, es un rasgo particular que, lejos de ser juzgado negativamente, debería ser comprendido en su justo contexto y especialmente valorado como la expresión de una disciplina con matices característicos y diferentes.

Una psicología eminentemente teorética y reflexiva no tiene por qué verse como necesariamente negativa, por más que al cimentarse con predominancia sobre esta condición deje marcado un rezago en su desarrollo como ciencia. Sin embargo, el énfasis en la teoría puede verse como un estadio necesario en la gestación de aproximaciones más orientadas a la investigación que, una vez presentes, habrán de constituirse sobre su base y cuya aparición se 
presume como parte de un desarrollo temporal posterior. En la psicología paraguaya no ha sucedido siempre eso, pero el rescate y revalorización de sus antecedentes olvidados y desconocidos, lejos de poseer un valor simple, emotivo o estrictamente testimonial y, por lo tanto, alejado de los objetivos reales que impelen al trabajo científico, mantiene el potencial de conducir hacia una evaluación critica de sus postulados $\mathrm{y}$, en consecuencia, hacia aquel demorado segundo momento que es el de la replicación.

Considerado en esta óptica particular, el trabajo de Ross (1915) publicado en Letras podría ser un eslabón en la síntesis del conocimiento actual de la psicología de la ciencia con los principios sustentados hace exactamente un siglo. Al respecto, y más allá de su interés histórico adventicio, cobra un nuevo sentido al penetrar en la mejor comprensión de algunos interrogantes antiguos pero de vigencia renovada.

\section{Referencias}

Báez, C. (1915). Filosofía política moderna. Letras, 1(2), 75-80.

Baldwin, J. M. (1913a). History of Psychology. A sketch and interpretation. Volume I. From the earliest times to John Locke. New York: G. P. Putnam's Sons.

Baldwin, J. M. (1913b). History of Psychology. A sketch and interpretation. Volume II. From John Locke to the present time. London: Watts \& Co.

Balfour, A. J. (1908). Decadence. Cambridge: Cambridge University Press.

Brett, G. S. (1912). A History of Psychology. Ancient and patristic. London: George Allen \& Company. http://dx.doi.org/10.1037/10579-000

Bucknill, J. C. (1859). The psychology of Shakespeare. London: Longman, Brown, Green, Longmans \& Roberts.

Cacciopo, J. T., \& Freberg, L. A. (2013). Discovering psychology. The science of mind. Belmont: Wadsworth.

Candolle, A. (1873). Histoire des sciences et des savants depuis deux siècles. Genève: H. Georg. 
Csikszentmihalyi, M. (2014). The systems model of creativity. The collected works of Mihaly Csikszentmihalyi. Dordrecht: Springer.

Domínguez, M. (1915). La Nación. Letras, 1(6), 295-304.

Dumont, F. (2010). A history of Personality Psychology. Theory, science and research from Hellenism to the twenty-first century. New York: Cambridge University Press. http://dx.doi.org/10.1017/CBO9780511676093

Feist, G. J. (1998). A meta-analysis of personality in scientific and artistic creativity. Personality and Social Psychology Review, 2(4), 290-309. http://dx.doi.org/10.1207/s15327957pspr0204_5

Feist, G. J. (2006). The Psychology of Science and the origins of the scientific mind. New Heaven: Yale University Press.

Feist, G. J. (2010). The function of personality in creativity. The nature and nurture of the creative personality. En J. C. Kaufman \& R. J. Sternberg (Eds.), The Cambridge Handbook of Creativity (pp. 113-130). New York: Cambridge University Press. http://dx.doi.org/10.1017/ cbo9780511763205.009

Feist, G. J. (2011). Psychology of science as a new subdiscipline in psychology. Current directions in Psychological Science, 20(5), 330334. http://dx.doi.org/10.1177/0963721411418471

Feist, G. J. (2012). The psychology of science is off and running but where do we go from here? En R. W. Proctor \& E. J. Capaldi (Eds.), Psychology of science: Implicit and explicit processes (pp. 13-39). New York: Oxford University Press. http://dx.doi.org/10.1093/acprof:o so/9780199753628.003.0001

Feist, G. J., \& Gorman, M. E. (2013). Introduction: Another brick in the wall. En G. J. Feist \& M. E. Gorman (Eds.), Handbook of the psychology of science (pp. 3-19). New York: Spinger.

Fine, G. (2014). The possibility of inquiry. Meno's paradox from Socrates to Sextus. New York: Oxford University Press. http://dx.doi.org/10.1093/ acprof:oso/9780199577392.001.0001

Galton, F. (1869). Hereditary genius. An inquiry into its laws and consequences. London: Macmillan. http://dx.doi.org/10.1037/ 13474-000 
Galton, F. (1874). English men of science: Their nature and nurture. London: Macmillan.

García, J. E. (2007). La psicología en Paraguay y el problema de la determinación de los pioneros. Revista Intercontinental de Psicología y Educación, Tercera Época, 9(2), 113-146.

García, J. E. (2009). Breve historia de la psicología en Paraguay. Psicología para América Latina, $N^{o}$ 17, Agosto 2009. Recuperado de http://www. psicolatina.org

García, J. E. (2014a). Eventos y protagonistas centrales para la historia de la psicología en el Paraguay. En G. Salas (Ed.), Historia de la Psicología en América del Sur. Diálogos y perspectivas (pp. 142-169). La Serena: Nueva Mirada Ediciones.

García, J. E. (2014b). La creación de soportes adecuados en la promoción de científicos e investigadores psicológicos. Cuadernos Pedagógicos, Octubre 2014, 37-46.

García, J. E. (2014c). Publicaciones psicológicas en la Revista del Instituto Paraguayo. Universitas Psychologica, 13(5), 1815-1833. Recuperado de http://revistas.javeriana.edu.co/sitio/psychologica

García, J. E. (2015a). Las brechas del pensamiento en la historia de la psicología. Arandu-UTIC, Revista Cientifica Internacional, 2(1), 29-73.

García, J. E. (2015b). Fundamentos históricos de la Psicología Educacional. En D. Jáuregui Camasca, R. León Donayre \& M. A. Rodríguez Rea (Eds.), Homenaje a Reynaldo Alarcón (pp. 393-426). Lima: Universidad Ricardo Palma, Editorial Universitaria.

García, J. E. (2015c). La revista Letras y su contribución a la psicología en el Paraguay. Revista Peruana de Psicología y Trabajo Social. En prensa.

Gattei, S. (2009). Karl Popper's philosophy of science. New York: Routledge. Hilgard, E. R., Leary, D. E., \& McGuire, G. R. (1991). History of psychology: A survey and critical assessment. Annual Review of Psychology, 42, 79107. http://dx.doi.org/10.1146/annurev.ps.42.020191.000455

Hoyningen-Huene, P. (1993). Thomas S. Kuhn's philosophy of science. Chicago: The University of Chicago Press. 
Ingenieros, J. (1915a). Las ideas sociológicas de Sarmiento. Letras, 1(2), 64-72.

Ingenieros, J. (1915b). Las ideas sociológicas de Sarmiento (Conclusión). Letras, 1(3), 121-128.

Klemm, O. (1914). A History of Psychology. New York: Charles Scibner's Sons. http://dx.doi.org/10.1037/10849-000

Lang, P. (2007). Schoenberg, Wittgenstein and the Vienna Circle. Bern: International Academic Publishers.

Lee, R. (2002). The eureka! moment. 100 key scientific discoveries of the $20^{\text {th }}$ century. New York: Routledge.

Lombroso, C. (1891). The man of genius. London: Walter Scott.

Lynn, R., \& Vanhanen, T. (2002). IQ and the wealth of nations. Westport: Praeger.

Mercante, V. (1915). Orientación de las cátedras de Filosofía. Letras, 1(2), 73-74.

Mercier, C. (1918). The origins of contemporary psychology. New York: P. J. Kenedy \& Sons.

Millon, T. (2004). Masters of the mind: Exploring the story of mental illness from ancient times to the new millennium. Hoboken: John Wiley \& Sons.

Okasha, S. (2002). Philosophy of science. A very short introduction. New York: Oxford University Press. http://dx.doi.org/10.1093/ actrade/9780198745587.001.0001

Owens, R. L. (2009). Genetics of creativity. En B. Kerr (Ed.), Encyclopedia of Giftedness, Creativity, and Talent (pp. 372-373). Thousand Oaks: Sage. http://dx.doi.org/10.4135/9781412971959.n163

Pérez Ríos, J. (1990). El análisis histórico en psicología como un proceso complejo. Revista de Historia de la Psicología, 11(3-4), 491-503.

Poincaré, H. (1918). Science and Method. London: Thomas Nelson \& Sons.

Rand, B. (1912). The classical psychologists. Selections illustrating psychology from Anaxagoras to Wundt. Boston: Houghton Mifflin Company. http://dx.doi.org/10.1037/10885-000 
Riquelme, M. (1948). Lecciones de Psicología (9ª ed.). Buenos Aires: Ángel Estrada Editores (edición original 1936).

Ross, R. (1915). El genio de la ciencia. Letras, 1(1), 33-35.

Sarton, G. (1948). The life of science. Essays in the history of civilization. New York: Henry Schuman.

Simonton, D. K. (1988). Scientific genius: A psychology of science. New York: Cambridge University Press.

Simonton, D. K. (1999a). Origins of genius. Darwinian perspectives on creativity. New York: Oxford University Press.

Simonton, D. K. (1999b). Creativity as blind variation and selective retention: Is the creative process Darwinian? Psychological Inquiry, 10(3), 309328 .

Simonton, D. K. (2009). Applying the Psychology of Science to the science of Psychology. Can psychologists use psychological science to enhance psychology as a science? Perspectives on Psychological Science, 4(1), 2-4. http://dx.doi.org/10.1111/j.1745-6924.2009.01093.x

Simonton, D. K. (2014). The Wiley Handbook of Genius. Chichester: Wiley Blackwell. http://dx.doi.org/10.1002/9781118367377

Sternberg, R. J. (1997). Thinking styles. New York: Cambridge University Press.

Tuckman, B. W., \& Monetti, D. M. (2011). Educational Psychology. Belmont: Wadsworth - Cengage Learning.

Uttal, W. R. (2004). Dualism. The original sin of cognitivism. Mahwah: Lawrence Erlbaum Associates.

Zhang, L. F., \& Sternberg, R. J. (Eds.) (2009). Perspectives on the nature of intellectual styles. New York: Springer. 